\title{
Acessos endoscópicos para lesões hipofisárias
}

\author{
Edson Flavio Lizarazu Oroz', Eberval Gadelha Figueiredo², \\ Fabio de Rezende Pinna ${ }^{3}$, Anderson Rodrigo Souza 4 , Maria Luana \\ Carvalho Viegas ${ }^{5}$, Daniella Brito Rodrigues ${ }^{6}$, Manoel Jacobsen Teixeira ${ }^{7}$
}

Hospital das Clínicas de La Paz, Bolívia, e Hospital das Clínicas da Faculdade de Medicina da Universidade de São Paulo, São Paulo, SP, Brasil.

\section{RESUMO}

Ao longo dos anos, a cirurgia minimamente invasiva avançou com a contribuição importante dos neurocirurgiões sobre a melhor opção para a abordagem terapêutica de tumores da região selar, partindo-se de uma cirurgia extremamente prejudicial, com alta morbidade e mortalidade, até a evolução de técnicas mais modernas, as quais dispõem de materiais mais adequados, que permitem ressecções completas com menor agressão às estruturas neurovasculares. Para praticá-las, é necessário um grupo de trabalho que envolva diferentes especialidades, como endocrinologia, oftalmologia, otorrinolaringologia, neurocirurgia e radioterapia, a fim de oferecer aos pacientes as melhores opções, garantindo atendimento individualizado, resolutivo e pouco agressivo. Há necessidade de um planejamento pré-operatório cuidadoso, com o uso de exames complementares avançados como ressonância magnética, tomografia computadorizada dos seios da face, dosagem sérica dos hormônios hipofisários e campimetria visual. Ainda, durante o procedimento cirúrgico, pode-se dispor de neuronavegação, tornando a cirurgia mais segura, especialmente nas reoperações. Por essas vantagens, a cirurgia transesfenoidal por microscopia convencional e por endoscopia é o procedimento atual de escolha para tratar tumores selares e fornecer adequada descompressão de estruturas neurais, com recuperação favorável mais rápida no pós-operatório em comparação com a abordagem transcraniana convencional.

\section{PALAVRAS-CHAVE}

Endoscopia, hipófise, neoplasias hipofisárias, hipofisectomia.

\section{ABSTRACT}

\section{Endoscopic approach of the pituitary lesions}

Over the years, minimally invasive surgery has advanced with the important contribution of neurosurgeons for the best option for the therapeutic approach of sellar tumors. Starting from an extremely harmful surgery with high morbidity and mortality until the evolution of techniques modern, which have the most suitable materials, which allow complete resections with less aggression to the neurovascular structures. To do them, is necessary a working group involving different specialities, such as endocrinology, ophthalmology, otolaryngology, neurosurgery and radiotherapy in order to offer patients the best options, ensuring individualized care, resolute and less aggressive. It's necessary a careful preoperative planning, using advanced exams such as MRI, CT scan of the sinuses, serum levels of pituitary hormones and visual perimetry. Still, the surgical procedure can be increased with the neuronavigation, making surgery more safer, especially in reoperations. Thus, because of these advantages, transphenoidal surgery for conventional microscopy and for endoscopy is the current procedure of choice for treating parasellar tumors, and provide adequate decompression of the neural structures with a favorable and faster recovery after surgery compared to the approach transcranial conventional.

\section{KEYWORDS}

Endoscopy, pituitary gland, pituitary neoplasms, hypophysectomy.

1 Neurocirurgião do Hospital de Clínicas de La Paz, Bolívia.

2 Supervisor da Divisão de Clínica Neurocirúrgica e chefe do Grupo de Neurocirurgia Vascular do Hospital das Clínicas da Universidade de São Paulo (USP), São Paulo, SP, Brasil.

3 Divisão de Otorrinolaringologia do Hospital das Clínicas da USP, São Paulo, SP, Brasil.

4 Neurocirurgião do Hospital das Clínicas da USP, São Paulo, SP, Brasil.

5 Acadêmica de Medicina da Faculdade de Medicina da Universidade Federal do Pará (UFPA), Belém, PA, Brasil.

6 Acadêmica de Medicina da Universidade do Estado do Pará (UEPA), Belém, PA, Brasil.

7 Diretor da Divisão de Clínica Neurocirúrgica do Hospital das Clínicas e professor titular da Disciplina de Neurocirurgia da USP, SP, Brasil. 


\section{Introdução}

Os tumores hipofisários constituem $10 \%$ de todos os tumores intracranianos e são geralmente benignos. ${ }^{1}$ A cirurgia minimamente invasiva da região selar foi amplamente estudada ao longo dos anos, por causa da peculiaridade anatômica da região, disposta profundamente na base do crânio e intimamente relacionada com estruturas neurovasculares importantes do encéfalo. Há, portanto, grande interesse pela forma mais eficaz e menos invasiva de resolver patologias da região selar e parasselar. ${ }^{2} \mathrm{O}$ uso da endoscopia nessas cirurgias tem condições favoráveis, em virtude da pouca resistência que a cavidade nasal e os seios esfenoidais oferecem, e desse modo constitui um atalho para a abordagem cirúrgica e permite boa ressecabilidade com menor taxa de complicações intra e pós-operatórias.

\section{História}

A história da Neurocirurgia de abordagem transesfenoidal iniciou-se com David Giordano no final do século XIX, a partir de estudos exaustivos em cadáveres, e prosseguiu com a realização da primeira cirurgia por via etmoidal e transesfenoidal para adenoma hipofisário, realizada em 1907 pelo neurocirurgião Scloffer. ${ }^{3,4}$ O procedimento foi um sucesso, porém com resultados estéticos desfavoráveis, razão pela qual foi alterado por Kocher, ${ }^{5}$ e finalmente a cirurgia foi orientada para a abordagem trans-septal sublabial. ${ }^{5}$ Entre 1910-1925, a técnica se difundiu na Academia Americana de Neurocirurgia graças às influências de Cushing, que praticava uma abordagem transesfenoidal a partir da modificação das técnicas descritas por Kocher-Scloffer. Entretanto, o método foi abandonado nas décadas seguintes em detrimento da abordagem transcraniana por via frontal, que permitia melhor descompressão do nervo óptico. $\mathrm{O}$ acesso transesfenoidal foi retomado em 1960, quando foi introduzido o auxílio da microscopia, segundo proposto por neurocirurgiões na América do Norte como Dott. ${ }^{6} \mathrm{Na}$ França, foi introduzido o uso de fluoroscopia; também foi o primeiro lugar a se usar um endoscópio para orientar o acesso nasal, considerado o primeiro avanço para endoscopia em cirurgia da região selar. ${ }^{6}$

O neurocirurgião vienense Hardy ${ }^{7}$ melhorou a técnica microcirúrgica que é usada até a atualidade, com algumas modificações que resultaram em baixas taxas de morbidade e mortalidade associadas, tendo contribuído, ainda, para o desenvolvimento dela com inovação de material específico. ${ }^{7}$ Em paralelo, há uma clara evolução na cirurgia endoscópica a partir dos avanços feitos por
Dandy, ${ }^{8}$ por meio da adição das técnicas descritas por Yaniv e Rappaport, ${ }^{9}$ no acesso transnasal combinado bilateral em direção ao seio esfenoidal.

A atual tendência é continuar o aperfeiçoamento da abordagem de modo a causar menor agressão e a melhorar a exposição, descrevendo pontos anatômicos da região esfenoidal e selar que garantam bom acesso à hipófise e reparação cirúrgica adequada de modo a minimizar erros durante a cirurgia. ${ }^{10}$

\section{Conceitos atuais}

Atualmente estão disponíveis endoscópios rígidos e flexíveis, que têm cabo de fibra óptica e fonte de luz LED integrada a uma fonte com ângulo óptico de $0^{\circ}, 30^{\circ}$ e $45^{\circ}$. Um dos endoscópios mais modernos disponíveis no mercado oferece o CCD (chip-on-the -point), que é um aperfeiçoamento do endoscópio de fibra óptica, por ter acoplado à ponta um chip CCD miniaturizado que desenha a imagem num monitor. A vantagem é uma melhor profundidade de campo. Não é necessário ser um foco de disparos subsequentes ou visão de curto do conjunto, uma vez que em ambos os casos a imagem tem sempre grande clareza. O uso da neuronavegação substitui radiografia transoperatória, ${ }^{11}$ sendo capaz de realizar um planejamento virtual, estimando com precisão a localização dessas lesões em três dimensões, reduzindo a morbidade e facilitando o acesso a áreas antes consideradas inseguras somente com o uso da ressonância magnética ou tomografia computadorizada. ${ }^{12}$

\section{Considerações pré-operatórias}

É essencial ter um conhecimento preciso da anatomia da região nasal e da base do crânio para evitar danos às principais estruturas vasculares adjacentes. Destarte, faz-se necessário um planejamento pré-operatório personalizado, o qual deve contemplar a análise de exames de imagem, especialmente a ressonância magnética (RM) com e sem gadolíneo, ${ }^{13}$ para delimitação precisa da massa tumoral, assim como para avaliação do grau de invasão do seio cavernoso (de acordo com a classificação de Knosp). ${ }^{14}$ Tomografia computadorizada (TC) de seios paranasais também é importante para a correta abordagem cirúrgica, atenuando dificuldades técnicas na identificação da linha média e do septo do seio esfenoidal. ${ }^{15}$

O exame oftalmológico é essencial, especialmente a fundoscopia e a análise do campo visual, em virtude 
dos frequentes déficits visuais decorrentes da compressão do quiasma óptico. As alterações do campo visual variam desde hemianopsia bitemporal até amaurose completa e constituem um importante fator prognóstico pós-cirúrgico. ${ }^{16}$

As alterações hormonais são frequentemente secundárias a tumores hipofisários e/ou procedimentos para o tratamento deles. ${ }^{17}$ A cirurgia pode ser curativa para alguns adenomas hiperfuncionantes, como o produtor de $\mathrm{GH},{ }^{18}$ enquanto outros tumores, como o prolactinoma, podem responder ao tratamento clínico com bromocriptina e cabergolina.

A patologia de base e o uso de medicações variam entre os pacientes e são fatores que interferem no resultado cirúrgico. A hipertrofia dos cornetos nasais em pacientes acromegálicos, assim como o uso de drogas inalatórias irritantes para a mucosa nasal, dificulta o acesso transnasal e determina maior sangramento intraoperatório. ${ }^{19}$

De modo geral, todos os casos devem ser avaliados individualmente; as alternativas terapêuticas devem considerar a idade do paciente, as características clínicas, os déficits neurológicos, as alterações hormonais e os aspectos anatômicos da lesão para, desse modo, evitar intercorrências e falhas de tratamento. ${ }^{20}$

\section{Procedimento cirúrgico endoscópico}

O endoscópio fornece uma visão panorâmica do seio esfenoidal, e isso permite que o cirurgião aborde essa região sem danificar estruturas adjacentes importantes, como as artérias carótidas e os nervos ópticos. ${ }^{10,12}$ A técnica foi adaptada ao longo dos anos, na procura de tratamentos cirúrgicos cada vez menos invasivos, de modo que melhores abordagens foram obtidas, permitindo movimentos mais amplos dos instrumentos endoscópicos. Desse modo, houve melhor visualização da cavidade e aumento da noção de profundidade e isso minimizou as limitações em relação às técnicas convencionais. $\mathrm{O}$ uso do espéculo nasal é controverso; alguns neurocirurgiões preferem não utilizá-lo; ${ }^{21}$ no entanto, o acesso transeptal requer descolamento de todo o septo e a utilização de espéculo nasal. $\mathrm{O}$ acesso endoscópico pode ser realizado por uma ou ambas as narinas; no acesso transnasal direto unilateral, o endoscópio e os instrumentos cirúrgicos são colocados pela mesma narina; e no acesso bilateral o endoscópio é colocado por uma narina e os instrumentos cirúrgicos pela outra. $\mathrm{O}$ acesso bilateral é mais utilizado, porque permite a adequada exposição cirúrgica sem perda de espaço para o manejo dos instrumentos. ${ }^{22}$
Antes do procedimento, deve-se explorar a cavidade nasal com um espéculo em busca do maior diâmetro, considerando a extensão e a possível lateralização tumoral, na dependência de seu tamanho e capacidade de invasão. Após antissepsia da região, o cirurgião deve introduzir gaze embebida com lidocaína e adrenalina a $1 \%$ em cada narina para causar vasoconstrição local. Por conseguinte, é necessário identificar e deslocar o corneto médio com uma cureta, para maior visibilidade; deve-se buscar o recesso esfenoetmoidal, que teoricamente fica a $1,5 \mathrm{~cm}$ da coana, em que se identificará o óstio esfenoidal, por onde será possível o acesso ao seio esfenoidal. Nessa fase, utiliza-se o endoscópio rígido com uma fibra óptica de $0^{\circ}$, e o vômer é removido para melhor visualização; prossegue-se, então, com a abertura do seio esfenoidal, que pode ser realizada com um drill pneumático. Uma vez dentro da mucosa sinusal, devem ser identificados os principais marcos anatômicos: $\mathrm{o}$ clivus inferiormente, as artérias carótidas de ambos os lados dos nervos ópticos e o recesso óptico-carotídeo. Nessa fase é possível usar a outra narina para a introdução do endoscópio e, novamente, utilizar o drill pneumático para a abertura da sela. Em seguida, a dura-máter pode ser aberta em X ou C, de acordo com a preferência do cirurgião; após a abertura da duramáter, prossegue-se com a identificação da hipófise e remoção do tumor (Figura 1). Nessa fase, o ângulo do endoscópio pode ser modificado para $30^{\circ}$ ou $45^{\circ}$, permitindo a revisão da cavidade e a procura de tumor residual. Na síntese, verifica-se se há ausência de saída do liquor e, se houver fístula, a reparação deve ser feita com enxerto autógeno e com cola biológica e/ou fibrina. No pós-operatório, deve-se atentar para a ocorrência de distúrbios como diabetes insipidus e déficits neurológicos, principalmente os visuais. ${ }^{12}$

\section{Endoscopia x microscopia convencional}

Talvez uma das maiores vantagens da cirurgia microscópica convencional seja a visão 3D que ela fornece ao cirurgião, enquanto a endoscopia mostra uma imagem em 2D com a perda da sensação de profundidade, mas essa desvantagem é superada com a habilidade crescente que o cirurgião adquire com a repetição da técnica. $\mathrm{O}$ maior benefício fornecido pelo endoscópio é o de permitir uma visão geral e, portanto, mais ampliada das estruturas anatômicas adjacentes, em virtude da facilidade de ter um ângulo de $30^{\circ}$ ou $45^{\circ}$, o que permite garantir o acesso a áreas anteriormente inacessíveis, permitindo maior ressecção. ${ }^{23}$ 

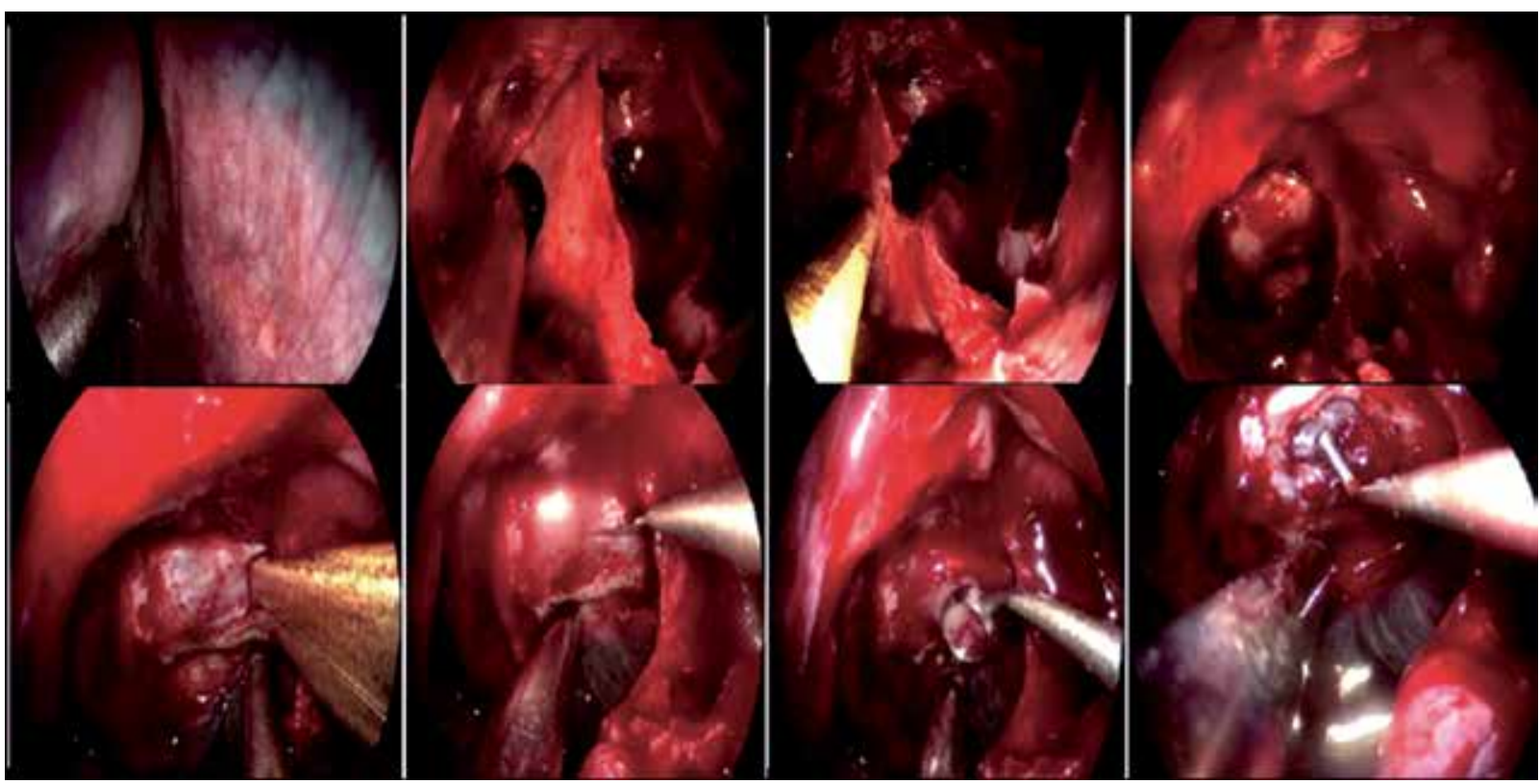

Figura 1 - Acesso endoscópico para lesões hipofisárias - Imagens intraoperatórias. Sequência do procedimento cirúrgico por via endoscópica para exérese de tumor hipofisário.

\section{Considerações finais}

A cirurgia minimamente invasiva é a técnica de escolha para o tratamento das patologias da região selar e, graças ao avanço da tecnologia e à experiência adquirida nos últimos anos, cada vez mais tem se mostrado um método seguro e capaz de garantir a ressecção de tumores que invadem o seio cavernoso ou o diafragma selar com o mínimo de dano às estruturas vasculares e ao mesmo tempo permitindo adequada descompressão do quiasma óptico.

As técnicas minimamente invasivas são mais toleradas no pós-operatório pelo paciente em relação às incisões convencionais de acesso transcraniano, permitindo um pós-operatório menos doloroso, o que garante um rápido regresso à sua atividade diária, com menor permanência hospitalar e menor morbidade nosocomial.

\section{Conflito de interesses}

Os autores declaram inexistência de conflito de interesses na realização deste estudo.

\section{Referências}

1. Laws ER, Jane JA Jr. Pituitary tumors - Long-term outcomes and expectations. Clin Neurosurg. 2001;48:306-19.
2. Musolino NRC. Tumores hipofisários: opções terapêuticas atuais e perspectivas futuras de tratamento. Arq Bras Endocrinol Metab. 2003;47(4):482-91.

3. Walker AE. A history of neurological surgery. New York: Hafner Publishing; 1967.

4. Artico M, Pastore FS, Fraioli B, Giuffrè R. The contribution of Davide Giordano (1864-1954) to pituitary surgery: the transglabellar-nasal approach. Neurosurgery. 1998;42(4):909-11.

5. Landolt AM. History of pituitary surgery from the technical aspect. Neurosurg Clin N Am. 2001;12(1):37-44.

6. Guiot J, Rougerie J, Fourestier M, Fournier A, Comoy C, Vulmiere J, et al. Intracranial endoscopic explorations. Presse Med. 1963;71:1225-8.

7. Hardy J. Surgery of the pituitary gland, using the transsphenoidal approach. Comparative study of 2 technical methods. Union Med Can. 1967;96(6):702-12.

8. Dandy WE. Extirpation of the choroid plexus of the lateral ventricles in communicating hydrocephalus. Ann Surg. 1918;68(6):569-79.

9. Yaniv E, Rappaport ZH. Endoscopic transseptal transsphenoidal surgery for pituitary tumors. Neurosurgery. 1997;40(5):944-6.

10. Campero A, Cruz J, Gonzales S. El punto esfenoselar: Ubicación anatómica y utilidad en 34 casos operados por vía endonasal transesfenoidal. Rev Arg Neuroc. 2006;20(1):7-12.

11. Basauri L, Concha Julio E. Neuronavegación I. Técnicas. Rev Med Clin Condes. 2000;11:119-24.

12. Brooks N, Badie B. Transsphenoidal approaches to the sella y suprasellar region. In: Badie B. Neurosurgical operative atlas: neuro oncology. $2^{\text {nd }}$ ed. New York: Theme; 2009. p. 3-8.

13. Eda M, Saeki N, Fujimoto N, Sunami K. Demonstration of the optic pathway in large pituitary adenoma on heavily T2 weighted MR images. Br J Neurosurg. 2002;16(1):21-9. 
14. Enseñat J, Ortega A, Topcewski T, Vilalta J, Obiols G, Mesa J, et al. Predictive value of the Knosp classification in grading the surgical resection of invasive pituitary macroadenomas. A prospective study of 23 cases. Neurocirurgia (Astur). 2006;17(6):519-26.

15. Terraes AR, De Los Reyes MR, Lagraña RA. Recalde F, Caceres C. Seno esfenoidal: cortes anatómicos. UNNE: Comunicaciones Científicas y Tecnológicas; 2000.

16. Girkin CA. Compressive optic neuropathy. In: Levin LA, Arnold AC, eds. Neuro-ophthalmology: the practical guide. New York, NY: Thieme Medical Publishers; 2005. p. 217-21.

17. Rosen T, Bengtsson BC. Epidemiology of adult onset hipopituitarism in Götenberg, Sweeden during 1056-1993. In: 10th International Congress of Endocrinology; 1996.

18. Jane JA Jr, Han J, Prevedello DM, Jagannathan J, Dumont AS, Laws ER Jr. Perspectives on endoscopic transsphenoidal surgery. Neurosurg Focus. 2005;19(6):E2.

19. Rivera J, Chavira M. Manejo anestésico del paciente consumidor de drogas. Rev Mex Anestesiolog. 2005;28(4):217-32.

20. Rojas D, Palma F, Wohllk N. Manejo de los adenomas hipofisiarios. Rev Chil Neuro-Psiquiat. 2008;46:140-7.
21. Nakao N, Nakai K, Itakura T. A minimally invasive endoscopic transsphenoidal approach with an endonasal septal pushover technique by using a modified nasal speculum. Minim Invasive Neurosurg. 2006;49(1):20-4.

22. De Divitiis E, Cappabianca P, eds. Endoscopic endonasal transsphenoidal surgery. New York: Springer-Verlag; 2003.

23. López Arbolay O, González González JL, Morales SO, Nedel VL. Cirugía transesfenoidal: primera opción de tratamiento para adenomas hipofisarios secretores de GH. Rev Cubana Endocrinol [revista en la Internet]. 2004. [citado 2013 Ago 12]; 15(3). Disponível em: <http:// scielo.sld.cu/scielo.php?script=sci_arttext\&pid=S1561$29532004000300003 \& \operatorname{lng}=$ es $>$.

\section{Endereço para correspondência:}

Edson Flavio Lizarazu Oroz

Rua das Magnolias III, casa A-8,

Cochabamba, Cercado, Bolívia

Telefones: 7054-9271/591 4441278

E-mail: edsonlizarazu@gnómica.com 